\title{
Estrutura das comunidades fitobentônicas do infralitoral da Baía de Sepetiba, RJ, Brasil
}

\author{
GILBERTO M. AMADO FILHO ${ }^{1,3}$, MARIA B.B.B. BARRETO², BIANCA V. MARINS', \\ CLAUDIA FELIX ${ }^{1}$ e RENATA P. REIS ${ }^{1}$
}

(recebido: 15 de maio de 2002; aceito: 28 de maio de 2003)

\begin{abstract}
Sublittoral phytobenthic community structure of Sepetiba Bay, RJ, Brazil). A description of the phytobenthic community structure in five sites of Sepetiba Bay, RJ, is presented. Sampling was done in the four seasons of the year 1999 (January, May, August, November), from sites at the inner bay region, directly subjected to the local anthropic impacts, to sites at the region close to the open sea, where the human interference is reduced. Destructive sampling method was used. Comparisons were based on the following parameters: species number, total biomass, diversity (H') and equitability (J). Ninety six taxa were identified: 61 rhodophytes, 18 chlorophytes, 15 phaeophytes and two cyanophytes. The highest number of taxa (65) and highest biomass values $\left(492.4 \mathrm{~g} . \mathrm{m}^{-2}\right)$ were found at site 5, located on Marambaia point. An increase in the diversity was seen from the inner bay to the open ocean region. Considering the biomass, Sargassum spp. were the most important macroalgae, followed by Padina gymnospora and other algae shorter in size as Hypnea spp., Gelidium pusillum, Pterocladiella caerulescens and Gelidiopsis spp. The utilization of the destructive sampling method allowed a detailed characterization of the composition and structure of Sepetiba Bay phytobenthic community, facilitating on the future, the identification of changes imposed to the algal community by using more simple methodological approaches.
\end{abstract}

Key words - biomass, diversity, macroalgae, pollution gradient

RESUMO - (Estrutura das comunidades fitobentônicas do infralitoral da Baía de Sepetiba, RJ, Brasil). Caracteriza-se neste estudo a estrutura das comunidades fitobentônicas em cinco locais da baía de Sepetiba, RJ. Foram realizadas amostragens do tipo destrutiva desde a região mais interna da baía, sujeita diretamente aos impactos antrópicos locais, até a região próxima ao mar aberto, onde a interferência humana é reduzida, em quatro épocas do ano de 1999 (janeiro, maio, agosto e novembro). Para comparação dos resultados foram utilizados: o número total de espécies, a biomassa total, a diversidade (H') e a equitabilidade (J). Foram identificados 96 táxons, sendo 61 rodofíceas, 18 clorofíceas, 15 feofíceas e duas cianofíceas. O maior número de táxons (65) e os mais elevados valores de biomassa $\left(492,4 \mathrm{~g} \cdot \mathrm{m}^{-2}\right)$ foram observados no ponto 5 , situado na Ponta da Marambaia. Ficou evidenciado o aumento da diversidade da região mais interna da baía em relação ao local mais próximo ao oceano. Sargassum spp. apresentaram a maior biomassa, seguida por Padina gymnospora e outras algas de menor porte como Hypnea spp., Gelidium pusillum, Pterocladiella caerulescens e Gelidiopsis spp. A utilização do método destrutivo permitiu uma caracterização detalhada da composição e da estrutura das comunidades fitobentônicas da baía de Sepetiba, facilitando no futuro, a identificação das mudanças impostas às comunidades através de abordagens metodológicas mais simples.

Palavras-chave - biomassa, diversidade, gradiente de poluição, macroalgas

\section{Introdução}

A baía de Sepetiba (BS), que até a década de 60 caracterizava-se pela pesca e pelo turismo, nos últimos trinta anos sofreu as conseqüências da expansão da região metropolitana do Rio de Janeiro, que resultou em grandes modificações nas estruturas espaciais,

1. Instituto de Pesquisas Jardim Botânico do Rio de Janeiro, Programa Zona Costeira, Rua Pacheco Leão 915, 22460-030 Rio de Janeiro, RJ, Brasil.

2. Universidade Federal Rural do Rio de Janeiro, Instituto de Biologia, Departamento de Botânica, Antiga Rodovia Rio São Paulo Km 47, 23851-970 Seropédica, RJ, Brasil.

3. Autor para correspondência: gfilho@jbrj.gov.br socio-econômicas e ecológicas da área em questão. Com o desenvolvimento industrial e adensamento dos núcleos urbanos, a BS tornou-se o segundo principal corpo receptor de efluentes industriais do Estado, principalmente de metais pesados derivados da indústria mínero-metalúrgica e, mais recentemente, de despejos urbanos (Amado Filho et al. 1999a). Além desses problemas, deve-se acrescentar a recente ampliação e modernização do Porto de Sepetiba, transformando-o em um super porto, concentrador de carga da costa sudeste da América do Sul ("hub port").

Por outro lado, apesar de toda sua importância ecológica, turística e pesqueira, são poucos os resultados obtidos nos últimos 30 anos sobre a diversidade da biota marinha da BS. Dentre os poucos estudos existentes 
deve-se destacar os esforços de grupos isolados como os de: Araujo e colaboradores (Araujo et al. 1997, 1999) que vem estudando a fauna ictiológica local. Neste contexto, fica evidente a escassa informação disponível e a necessidade urgente de ações no sentido de se conhecer a diversidade das comunidades marinhas da $\mathrm{BS}$, antes que o processo de degradação se torne irreversível. Deve-se ressaltar ainda, que a BS foi recentemente escolhida como área piloto no Brasil para a execução do projeto "Remoção de barreiras para a implementação efetiva do controle de água de lastro e medidas de gestão em países em desenvolvimento" (www.globallast.imo.org), iniciativa da Organização Marítima Internacional (IMO) em associação com o Programa das Nações Unidas para o Desenvolvimento (UNDP) e o Fundo para o Meio Ambiente Mundial (GEF).

As macroalgas marinhas são responsáveis por até $68 \%$ da cobertura do substrato de costões rochosos da BS (Amado Filho et al. 1999b) e a exceção dos estudos florísticos realizados no início da década de 80 (Pedrini 1980), pouco se conhece sobre a estrutura e dinâmica das comunidades bentônicas da BS. Széchy (1996) apresenta uma caracterização de flora e fauna associadas à Sargassum cymosum C. Agardh var. cymosum em um ponto situado no limite oeste (Ibicuí) da BS. Reis \& Yoneshigue-Valentin (1998) apresentam uma caracterização da variação sazonal da biomassa e da flora acompanhante de Hypnea musciformis (Wulfen) J. V. Lamour. em uma localidade no município de Búzios e duas localidades na BS (em Ibicuí e na Ilha de Itacuruça).

Com o intuito de gerar subsídios para futuros programas de monitoramento, é descrita neste trabalho a estrutura das comunidades fitobentônicas do infralitoral de cinco locais da baía de Sepetiba.

\section{Material e métodos}

Foram amostrados cinco pontos (figura 1) escolhidos de modo a representar todas as regiões da baía, e estabelecer um possível gradiente, da região mais interna da baía, sujeita diretamente aos impactos antrópicos (Amado Filho et al. 1999a) à região próxima ao mar aberto, onde a interferência humana é menor.

As amostragens foram realizadas em janeiro (verão), maio (outono), agosto (inverno) e novembro (primavera), durante o ano de 1999 (temperatura do ar média anual de $22,4{ }^{\circ} \mathrm{C}$ e precipitação média anual de $1.383,9 \mathrm{~mm} \cdot$ ano $^{-1}$, INMET) a $1 \mathrm{~m}$ abaixo da linha da maré mais baixa (maré $0,0 \mathrm{~m}$, tábua de marés, DHN/MM). Esta profundidade foi escolhida por apresentar a maior abundância de macroalgas
(Amado Filho et al. 1999b). Uma trena com 20 m de extensão foi posicionada ao longo de uma transecção horizontal paralela a linha da água, e ao longo desta trena 5 quadrados de $20 \times 20 \mathrm{~cm}$ foram dispostos aleatoriamente (utilizou-se uma tabela de números aleatórios). De modo a uniformizar as comparações entre os locais, foi escolhida para os 5 pontos, uma faixa do costão com inclinação igual ou inferior a 20 graus. Todos os organismos contidos em cada quadrado foram retirados cuidadosamente e acondicionados em solução de formaldeido a $4 \%$. As amostragens foram realizadas através de mergulho autônomo.

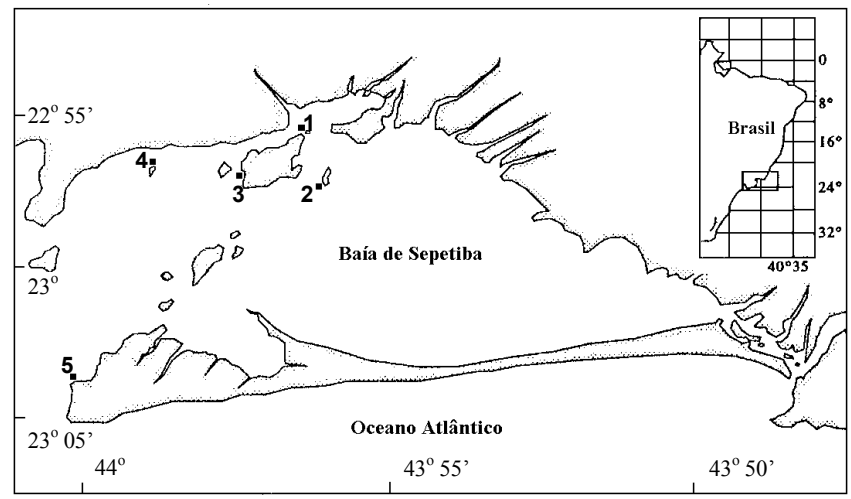

Figura 1. Localização dos cinco pontos de amostragens na baía de Sepetiba: 1 - Ilha do Gato; 2 - Ilha do Martins; 3 - Praia Grande; 4 - Ilha Duas Irmãs; 5 - Ponta da Marambaia.

Figure 1. Localization of the five sampling sites at Sepetiba Bay: 1- Gato Island; 2 - Martins Island; 3 - Grande Beach; 4 - Duas Irmãs Island; 5 - Marambaia Point.

No laboratório, foi realizada a identificação taxonômica, quantificação da biomassa, esta última através de secagem em estufa a $60^{\circ} \mathrm{C}$ até peso constante. Para as espécies que não atingiram a massa mínima $(0,001 \mathrm{~g})$, atribuiu-se o valor arbitrário de $0,0001 \mathrm{~g}$.

Os resultados foram comparados quanto ao número total de espécies, biomassa total, diversidade de Shannon-Wiener (H') e equitabilidade de Pielou (J) (Brower et al. 1997). A análise de variância (ANOVA) bi-fatorial ou uni-fatorial seguida pelo teste de "Tukey" foi utilizada para avaliar a significância entre diferenças na biomassa, diversidade e equitabilidade total entre os locais (Zar 1996). A homogeneidade das variâncias foi verificada pelo teste de Cochran (Zar 1996, Winer 1971) e foi assumido que os dados são independentes (Zar 1996). Estas análises são consideradas robustas para desvios da normalidade (Underwood 1981, Zar 1996).

\section{Resultados}

Foram identificados 96 táxons, sendo 61 rodofíceas, 18 clorofíceas, 15 feofíceas e duas cianofíceas (tabela 1). O local que apresentou o maior 
número de táxons (65) foi o ponto 5 , situado na Ponta da Marambaia e, o que apresentou menor número (39) foi o ponto 1, Ilha do Gato. Do total de táxons, 17 foram comuns a todos os pontos de amostragem e 31 estiveram restritos a apenas um, destacando-se o ponto 5 onde foram encontrados 17 táxons exclusivos.

Não foi observada tendência geral de variação no número de táxons ao longo do ano ou uma época preferencial para ocorrência de maior número de táxons entre os locais de coleta (figura 2). Apenas nos pontos 1 e 5 houve diminuição do número de táxons no outono e/ou inverno. No ponto 1 variou de 16 táxons no outono e inverno para 36 na primavera e, no ponto 5 , variou de 16 no outono para 50 no verão.

Em relação à biomassa total, observou-se uma variação da média total de 95,9 g.m $\mathrm{m}^{-2}$ no ponto 4 para 492,4 g. $\mathrm{m}^{-2}$ no ponto 5 (figura 3 ). Comparando-se a biomassa entre os pontos de coleta, destaca-se o ponto 5 , local que apresentou os valores mais elevados de biomassa e que foram significativamente diferentes (ANOVA bi-fatorial, teste de Tukey, $\mathrm{p}<0,05$ ) dos valores encontrados nos demais locais de coleta (ponto $1, \mathrm{p}=0,015$; ponto $2, \mathrm{p}=0,019$; ponto $4, \mathrm{p}=0,006$ ), exceto os do ponto $3(\mathrm{p}=0,137)$. Em termos sazonais, comparando-se (ANOVA bi-fatorial, teste de Tukey, $\mathrm{p}<0,05)$ a biomassa total média entre as quatro épocas do ano em cada local (figura 3 ) não foram encontradas diferenças significativas $(\mathrm{p}<0,05)$ nos pontos $1,2,3 \mathrm{e}$ 4. Apenas no ponto 5 foram encontradas diferenças significativas entre o verão e primavera, $p=0,02$; outono e inverno, $\mathrm{p}=0,02$; inverno e primavera, $\mathrm{p}=0,0008$.

A comparação dos índices de diversidade médios encontrados nos cinco pontos de coleta (figura 4) evidencia a tendência ao aumento da diversidade da região mais interna da baía (ponto $1, H^{\prime}=0,86 \pm 0,49$ ) em relação ao local mais próximo ao oceano (ponto 5 , $\left.\mathrm{H}^{\prime}=2,19 \pm 0,41\right)$. Esta tendência é confirmada por diferenças significativas detectadas entre o ponto $1 \mathrm{e}$ os pontos $3\left(\mathrm{H}^{\prime}=1,74 \pm 0,31, \mathrm{p}<0,03\right), 4\left(\mathrm{H}^{\prime}=1,73 \pm\right.$ $0,41, \mathrm{p}<0,03)$ e $5(\mathrm{p}<0,001)$ e do ponto $2\left(\mathrm{H}^{\prime}=1,35 \pm\right.$ $0,13)$ em relação ao ponto $5(\mathrm{p}<0,04)$. A mesma tendência foi encontrada quando analisados os resultados do índice de equitabilidade (figura 5); observa-se o aumento do valor do índice do ponto 1 para o ponto 5 e foram encontradas diferenças significativas entre o ponto $1(\mathrm{~J}=0,26 \pm 0,14)$ e os pontos $3(\mathrm{~J}=0,49 \pm 0,09, \mathrm{p}=0,027)$ e $5(\mathrm{~J}=0,65 \pm$ $0,06, p=0,005)$ e entre o ponto 3 e o $5(p=0,025)$. O resultado do índice de equitabilidade relativamente baixo encontrado no ponto 1 indica a contribuição de poucas espécies para a biomassa total encontrada.

Pode-se observar que o percentual de contribuição médio para a biomassa total de um único táxon (no caso das espécies de Sargassum agrupando-as como Sargassum spp.) foi sempre superior a $60 \%$ nos pontos 1 e 2, onde também foram encontrados os mais baixos índices de diversidade e equitabilidade. No ponto 1 , as espécies de Sargassum encontradas contribuíram com $62 \%\left(127,9\right.$ g. $\left.\mathrm{m}^{-2}\right)$ para a biomassa total no verão, sendo substituídas por P. gymnospora $\left(71 \%\right.$ - 67,5 g.m ${ }^{-2}$ no outono a $96 \%$ - 185,8 g.m. ${ }^{-2}$ no inverno) nas outras três épocas do ano (figura 6 , tabela 2 ). No ponto 2 , as espécies de Sargassum encontradas permaneceram como os organismos dominantes ao longo de todo o período amostral, tendo sua contribuição variado de $70,9 \%$ a $93,5 \%$ (figura 6 ). A biomassa média de Sargassum variou de 13,3 g.m $\mathrm{m}^{-2}$ no outono a 98,3 g.m $\mathrm{m}^{-2}$ na primavera (tabela 2 ).

A partir do ponto 3, não mais se observou a tendência de apenas um táxon contribuir com mais de $50 \%$ da biomassa total em todas as épocas do ano (figura 7, tabela 2). Verificou-se que no ponto 3, além de Sargassum spp. e P. gymnospora, H. musciformis $\left(71,6\right.$ g. $\left.\mathrm{m}^{-2}\right)$ e G. planicaulis $\left(52,7\right.$ g. $\left.\mathrm{m}^{-2}\right)$ contribuiram com até $26,7 \%$ e $20,1 \%$, respectivamente, da biomassa total. Neste local foram encontrados os valores mais elevados de biomassa média das espécies de Sargassum ao longo do ano, variando de 92,7 a 218,1 g.m ${ }^{-2}$ (tabela 2). No ponto 4, observou-se um padrão de composição e biomassa no período da primavera/verão, onde as espécies de Sargassum contribuíram com cerca de $70 \%$ da biomassa total e outro padrão no período do outono/inverno onde espécies de menor porte como $H$. spinella, G. pusillum e D. ciliolata, contribuíram de maneira semelhante a Sargassum spp. e P. gymnospora em termos percentuais para a biomassa total (figura 8 , tabela 2). No ponto 5 , a exceção de $C$. sertularioides que no outono contribuiu com 41,6\% $\left(239,9\right.$ g.m $\left.{ }^{-2}\right)$ da biomassa total, nenhuma outra espécie contribuiu com mais de $25 \%$ para a biomassa total. Neste local, pode-se verificar a participação de várias espécies (G. cervicornis, C. taylorii, G. marginata, S. schroderii, C. teedei, $D$. delicatula), dentre outras com valores de biomassa média semelhantes, contribuindo com normalmente entre 5\% e 15\% (figura 9) para a biomassa total local (tabela 2).

\section{Discussão}

Mesmo não tendo como objetivo principal realizar um inventário florístico, pôde-se verificar que 16 dos 
Tabela 1. Táxons encontrados na baía de Sepetiba e suas respectivas ocorrências nos cinco locais de amostragem.

Table 1. List of taxa found at Sepetiba Bay and their respective occurrence on the five studied sites.

\begin{tabular}{|c|c|c|c|c|c|}
\hline \multirow[t]{2}{*}{ Táxons } & \multicolumn{5}{|c|}{ Locais de amostragem } \\
\hline & 1 & 2 & 3 & 4 & 5 \\
\hline
\end{tabular}

CHLOROPHYTA(18)

Acetabularia calyculus J.V. Lamour.

Boodleopsis pusilla (Collins) Taylor, Joly \& Bernat.

Bryopsis pennata J.V. Lamour.

Bryopsis plumosa (Huds.) C. Agardh

Caulerpa fastigiata Mont.

Caulerpa sertularioides (S.G. Gmel.) M. Howe

Chaetomorpha aerea (Dillwyn) Kütz.

Chaetomorpha gracilis Kütz.

Cladophora brasiliana $\mathrm{G}$. Martens

Cladophora coelothrix Kütz.

Cladophora rupestris (L.) Kütz.

Cladophora vagabunda (L.) C. Hoek

Codium decorticatum (Woodw.) M. Howe

Codium taylorii P.C. Silva

Derbesia marina (Lyngb.) Solier

Enteromorpha flexuosa (Wulfen) J. Agardh subsp. flexuosa

Ulva lactuca L.

Ulva rigida C. Agardh

SUB-TOTAL

PHAEOPHYTA (15)

Bachelotia antillarum (Grunov) Gerloff

Colpomenia sinuosa (Roth) Derbès \& Solier

Dictyopteris delicatula J.V. Lamour.

Dictyota cervicornis Kütz.

Dictyota ciliolata Sond.ex Kütz.

Hincksia mitchelliae (Harv.) P.C. Silva

Padina gymnospora (Kütz.) Sond.

Padina tetrastromatica Hauck

Sargassum cymosum C. Agardh var. cymosum

Sargassum filipendula C. Agardh var. filipendula

Sargassum filipendula C. Agardh var. laxum J. Agardh

Sargassum stenophyllum Mart.

Sargassum vulgare C. Agardh var. vulgare

Spatoglossum schroederi (C. Agardh) Kütz.

Sphacelaria tribuloides Menegh.

SUB-TOTAL

RHODOPHYTA (61)

Acanthophora spicifera (Vahl) Borgesen

Achrochaetium microscopicum (Nägeli ex Kütz.) Nägeli

Aglaothamnion felliponei (M. Howe) Aponte, Ballant. \& Norris

Aglaothamnion uruguayense (Taylor) Aponte, Ballant \& Norris

Amphiroa beauvoisii J.V. Lamour.

Amphiroa brasiliana Decne.

Amphiroa fragilissima (L.) J.V. Lamour.

Anothrichium tenue (C. Agardh) Nägeli

Bostrychia radicans (Mont.) Mont.

\begin{tabular}{|c|c|c|c|c|}
\hline+ & - & - & - & - \\
\hline- & - & - & + & - \\
\hline+ & + & - & + & - \\
\hline+ & + & + & - & + \\
\hline- & - & - & - & + \\
\hline- & - & - & - & + \\
\hline+ & - & - & - & - \\
\hline- & - & - & + & - \\
\hline+ & - & - & + & + \\
\hline+ & + & - & - & + \\
\hline+ & + & + & + & + \\
\hline+ & + & + & + & - \\
\hline- & + & + & + & + \\
\hline+ & - & + & + & + \\
\hline- & + & + & - & - \\
\hline+ & + & + & + & + \\
\hline+ & - & + & + & + \\
\hline- & - & - & - & + \\
\hline 11 & 8 & 8 & 10 & 11 \\
\hline- & - & - & + & - \\
\hline- & - & + & - & + \\
\hline- & - & - & + & + \\
\hline- & - & - & + & - \\
\hline- & - & + & + & + \\
\hline- & + & - & + & + \\
\hline+ & + & + & + & + \\
\hline- & - & - & - & + \\
\hline- & + & + & + & + \\
\hline+ & - & - & - & - \\
\hline+ & + & + & - & - \\
\hline- & + & + & + & + \\
\hline- & + & + & - & - \\
\hline- & - & + & - & + \\
\hline- & - & - & - & + \\
\hline 3 & 6 & 8 & 7 & 10 \\
\hline- & - & + & + & - \\
\hline- & + & + & + & - \\
\hline - & + & + & + & - \\
\hline - & + & + & - & + \\
\hline- & - & + & + & + \\
\hline - & - & - & + & - \\
\hline - & - & - & - & + \\
\hline - & - & + & + & - \\
\hline - & - & - & - & + \\
\hline
\end{tabular}


Táxons Locais de amostragem

\begin{tabular}{lllll}
\multicolumn{6}{c}{ Locais de amostragem } \\
\hline 1 & 2 & 3 & 4 & 5
\end{tabular}

Bostrychia tenella (J.V. Lamour.) J. Agardh

Bryocladia thyrsigera (J. Agardh) F. Schmitz

Centroceras clavulatum (C. Agardh) Mont.

Ceramium brevizonatum Petersen var. caraibicum Pet. \& Borgesen

Ceramium deslongchampsii Chav. ex Duby

Ceramium diaphanum (Lightf.) Roth

Ceramium flaccidum (Kütz.) Ardiss.

Ceramium luetzelburgii O.C. Schmidt

Ceramium sp. 1

Ceramium sp. 2

Champia parvula (C. Agardh) Harv.

Champia salicornioides Harv.

Champia vieillardii Kütz.

Chondracanthus acicularis (Roth) Fredericq

Chondracanthus teedei (Mertens ex Roth) Fredericq

Dasya brasiliensis E.C. Oliveira \& Y. Braga

Erythrotrichia carnea (Dillwyn) J. Agardh

Galaxaura marginata (J. Ellis \& Sol.) J.V. Lamour.

Gelidiopsis planicaulis (W.R. Taylor) W.R. Taylor

Gelidiopsis variabilis (Grev. ex J. Agardh) F. Schmitz

Gelidium crinale (Turner) Gaillon

Gelidium pusillum (Stackh.) Le Jolis var. pusillum

Gracilaria caudata J. Agardh

Gracilaria cervicornis (Turner) J. Agardh

Gracilariopsis tenuifrons (Bird \& Oliveira) Fredericq \& Hommersand

Grateloupia doryphora (Mont.) M. Howe

Grateloupia filicina (J.V. Lamour.) C. Agardh

Gymnogongrus griffithsiae (Turner) Mart.

Halymenia elongata C. Agardh

Halymenia rosea Howe \& W.R. Taylor

Herposiphonia secunda (C. Agardh) Ambronn f. secunda

Herposiphonia secunda (C. Agardh) Ambronn f. tenella Wynne

Hypnea musciformis (Wulfen) J. V. Lamour.

Hypnea spinella (C. Agardh) Kützing

Jania adhaerens J.V. Lamour.

Jania capillacea Harv.

Polysiphonia denudata (Dillwyn) Grev. ex Harv.

Polysiphonia ferulacea Surh ex J. Agardh

Polysiphonia scopulorum Harv. var. villum (J. Agardh) Hollenb.

Polysiphonia sphaerocarpa Borgesen

Polysiphonia subtilissima Mont.

Polysiphonia tepida Hollenb.

Polysiphonia tongatensis Harv. ex Kütz.

Pterocladiella caerulescens (Kütz.) Santel. \& Hommers.

Pterosiphonia parasitica (H.) Falk. var. australis Joly \& Cord.-Marino

Pterosiphonia pennata (C. Agardh) Falkenb.

Rhodymenia pseudopalmata (J.V. Lamour.) P.C. Silva

Sahlingia subintegra (Rosenv.) Kornmann

Solieria filiformis (Kütz.) P.W. Gabrielson

\begin{tabular}{|c|c|c|c|c|}
\hline- & - & - & - & + \\
\hline- & - & - & - & + \\
\hline+ & + & + & + & + \\
\hline- & + & - & - & - \\
\hline- & - & + & + & - \\
\hline+ & + & - & + & + \\
\hline+ & + & + & + & + \\
\hline- & - & + & - & - \\
\hline+ & + & + & + & + \\
\hline+ & + & + & + & + \\
\hline- & - & + & + & + \\
\hline- & - & + & - & + \\
\hline- & - & - & + & - \\
\hline+ & + & + & + & + \\
\hline+ & + & + & + & - \\
\hline- & - & + & + & + \\
\hline+ & + & + & + & - \\
\hline- & - & - & - & + \\
\hline+ & + & + & + & + \\
\hline+ & + & + & + & + \\
\hline+ & + & + & + & + \\
\hline+ & + & + & + & + \\
\hline- & - & - & - & + \\
\hline- & - & + & + & + \\
\hline- & - & - & - & + \\
\hline- & - & + & - & + \\
\hline+ & + & - & + & + \\
\hline+ & + & + & + & - \\
\hline- & - & - & + & + \\
\hline- & - & - & - & + \\
\hline- & - & + & - & - \\
\hline- & - & - & - & + \\
\hline+ & - & + & + & + \\
\hline+ & + & + & + & + \\
\hline- & + & + & + & + \\
\hline- & + & + & + & - \\
\hline+ & + & + & + & - \\
\hline+ & + & + & + & + \\
\hline- & - & - & - & + \\
\hline- & - & - & - & + \\
\hline- & + & + & + & + \\
\hline+ & + & + & - & - \\
\hline+ & + & + & + & + \\
\hline+ & + & + & + & + \\
\hline- & - & - & + & + \\
\hline- & + & - & - & + \\
\hline- & - & - & - & + \\
\hline+ & + & + & + & + \\
\hline- & - & + & - & + \\
\hline
\end{tabular}


continuação

\begin{tabular}{|c|c|c|c|c|c|}
\hline \multirow{2}{*}{ Táxons } & \multicolumn{5}{|c|}{ Locais de amostragem } \\
\hline & 1 & 2 & 3 & 4 & 5 \\
\hline Spyridia filamentosa (Wulfen) Harv. & + & - & + & + & + \\
\hline Stylonema alsidii (Zanardini) K.M. Drew & + & + & + & + & - \\
\hline Wrangelia argus (Mont.) Mont. & - & - & - & + & - \\
\hline SUB-TOTAL & 24 & 30 & 40 & 40 & 43 \\
\hline \multicolumn{6}{|l|}{ CYANOPHYTA(2) } \\
\hline Lyngbya sp. & + & - & - & + & - \\
\hline Microcoleus sp. & - & - & + & + & + \\
\hline SUB-TOTAL & 1 & 1 & 2 & 1 & \\
\hline TOTAL & 39 & 44 & 57 & 60 & 65 \\
\hline
\end{tabular}

96 táxons identificados neste trabalho (16,6\%), ainda não haviam sido citados em estudos anteriores sobre o fitobentos da baía de Sepetiba (Mitchell et al. 1979, Pedrini 1980, Szechy 1996, Reis \& Yoneshigue-Valentin 1998). Destes 16 táxons, quatro são de clorofíceas, B. plumosa, C. brasiliana, C. coelothrix e D. marina, uma de feofícea, $P$. tetrastromatica e 11 de rodofíceas, A. tenue, Ceramium sp. 1, Ceramium sp. 2, G. caudata, G. tenuifrons, J. adhaerens, P. denudata, P. scopulorum, P. sphaerocarpa, P. tepida, P. tongatensis. Considerando-se, que a exceção de $P$. tetrastromatica, $G$. caudata, G. tenuifrons e $J$. adhaerens, as demais espécies são de pequeno porte, filamentosas e de rápido crescimento, sugerindo-se que algumas dessas espécies possam ser consideradas como introduções recentes na baía de Sepetiba. O transporte e a introdução de espécies aquáticas, intencional ou acidental, são atualmente considerados principais ameaças à biodiversidade (Lubchenco et al. 1991). Deve-se considerar que os

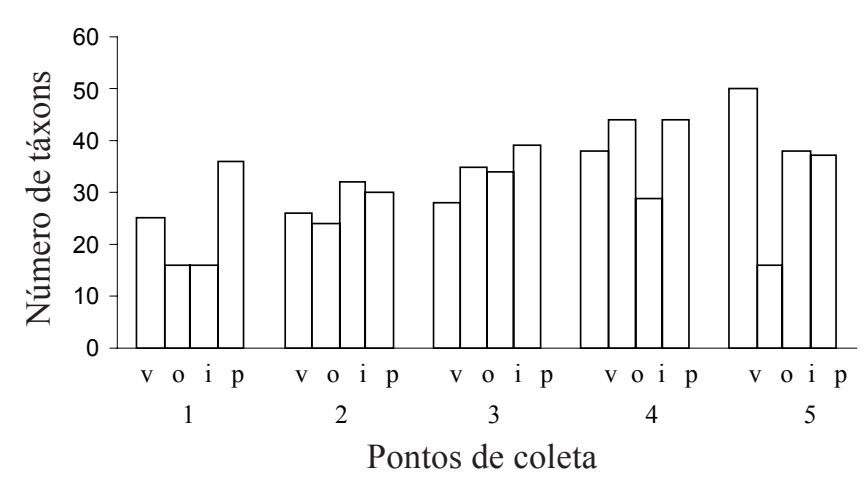

Figura 2. Número total de táxons encontrados nos cinco locais estudados: (v) verão, (o) outono, (i) inverno e (p) primavera.

Figure 2. Total number of taxa found in the five studied sites: (v) Summer, (o) Autumn. (i) Winter and (p) Spring. mecanismos de introdução, estabelecimento e invasão de uma espécie são análogos, mas possivelmente diferentes dos mecanismos de dispersão, competição e estabelecimento de espécies que ocorrem de maneira natural. Sendo assim, os resultados obtidos neste trabalho indicam a possibilidade da introdução recente de espécies de algas na baía de Sepetiba e servirão como base para a avaliação da diversidade da biota marinha local a ser realizada futuramente de acordo com o protocolo estabelecido pela Organização Marítima Internacional para o Programa Água de Lastro (Hewitt \& Martin 2001).

A avaliação espacial das comunidades fitobentônicas da BS nos cinco locais amostrados evidenciou um aumento do número de espécies, da biomassa total, e consequentemente dos parâmetros derivados, diversidade e equitabilidade, da região mais interna da baía (ponto 1) para a região próxima ao mar aberto (ponto 5). Pode-se relacionar este padrão de distribuição espacial à influência de fatores ambientais naturais, como salinidade, turbidez, movimentação da água e também a fatores de origem antrópica, como a concentração de metais pesados. Os dados obtidos por Barcellos (1995), Rees et al. (1998) e FEEMA/GTZ (2000) mostram que a região mais interna da baía (onde estão localizados os pontos 1 e 2) apresentou menores valores de salinidade (média de 27 a 30 psu), valores elevados de MPS (material particulado em suspensão $\left.>100 \mathrm{mg} \cdot \mathrm{l}^{-1}\right)$, menor circulação de massas d'agua (\% de argila no sedimento $>50$ ), e concentrações elevadas de metais pesados no sedimento superficial $\left(\mathrm{Zn}>350 \mu \mathrm{g} \cdot \mathrm{g}^{-1}\right.$ e Cd $\left.>3,0 \mu \mathrm{g} \cdot \mathrm{g}^{-1}\right)$. Por outro lado, numa situação oposta, está a região do ponto 5, situado na Ponta da Marambaia, que apresenta salinidade de $35 \mathrm{psu}$, MPS $<100 \mathrm{mg} . \mathrm{l}^{-1}$, $\%$ de argila no sedimento $<5$, e concentrações de $\mathrm{Zn}$ $<8,0 \mu \mathrm{g} \cdot \mathrm{g}^{-1}$ e $\mathrm{Cd}<0,2 \mu \mathrm{g} \cdot \mathrm{g}^{-1}$. 


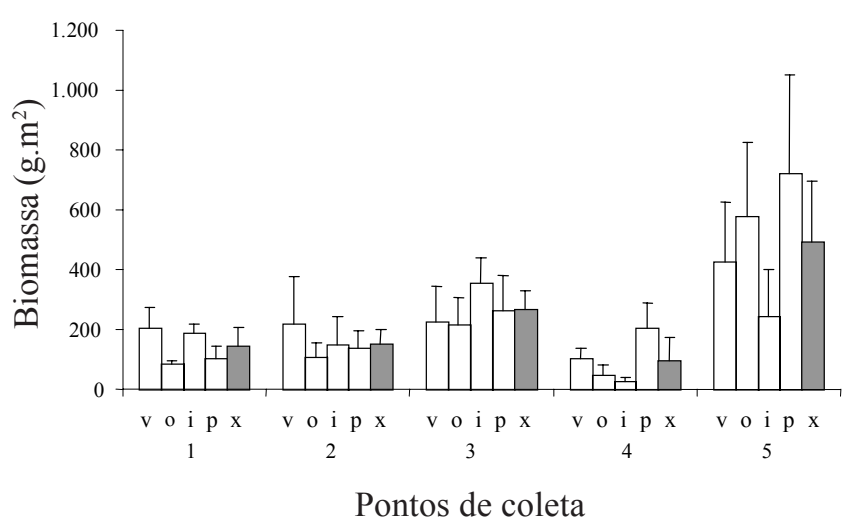

Figura 3. Biomassa média por $\mathrm{m}^{2}$ em cada época do ano (v) verão, (o) outono, (i) inverno e (p) primavera, e biomassa média anual por $\mathrm{m}^{2}(\mathrm{x})$ encontrada nos cinco locais estudados. Linha acima da barra $=$ desvio padrão.

Figure 3. Mean biomass per $\mathrm{m}^{2}$ in each season of the year, (v) Summer, (o) Autumn, (i) Winter and (p) Spring and mean year biomass per $\mathrm{m}^{2}(\mathrm{x})$ from the five studied sites.

Amado Filho et al. (1999b) em um estudo preliminar de avaliação do percentual de cobertura de macroalgas em dois locais da baía de Sepetiba, comentaram que a menor diversidade encontrada na Ilha do Gato (ponto 1), incluindo a ausência de rodofíceas, como H. musciformis, deve estar relacionada a maior disponibilidade de metais pesados nesta área e conseqüentemente, a níveis diferenciados de tolerância aos metais pesados apresentado pelas espécies de

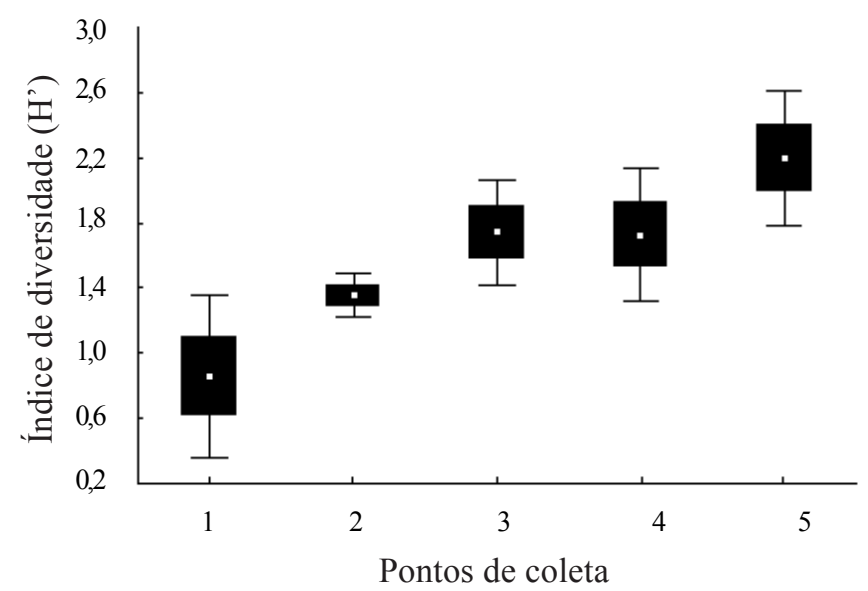

Figura 4. Representação gráfica dos valores do índice de diversidade (H') calculados para os cinco locais estudados. - média \pm erro padrão; I média \pm desvio padrão; $\square$ média.

Figure 4. Diversity index (H') calculated for the five studied sites. $\square$ mean \pm standard error; I mean \pm standard deviation; $\square$ mean. macroalgas. Diferentemente de outros locais sujeitos a um gradiente de poluição, como por exemplo as baías de Guanabara (Teixeira et al. 1987) e de Santos (Berchez \& Oliveira 1992) onde os locais mais contaminados são dominados por clorofíceas oportunistas como Ulva, Enteromorpha ou Cladophora, na Baía de Sepetiba, os locais onde as concentrações dos metais Zn e Cd são mais elevadas (pontos 1 e 2), são dominados pelas feofíceas, Sargassum spp. e $P$. gymnospora. Este padrão encontrado na baía de Sepetiba pode ser explicado pela maior tolerância de Padina e Sargassum a concentrações elevadas de metais pesados quando comparadas com rodofíceas e clorofíceas (Amado Filho et al. 1997a, Engdahl et al. 1998, Markham et al. 1980, Murugadas et al. 1995). Isto indica, também, a ausência de poluição por hidrocarbonetos em níveis que afetam processos reprodutivos de algas pardas (Jaenicke 1977), como foi sugerido para a baía de Guanabara (Teixeira et al. 1987).

A análise dos resultados de biomassa das espécies encontradas evidencia a importância das espécies de Sargassum nas comunidades fitobentônicas da baía de Sepetiba e vem reforçar a afirmação de que o gênero Sargassum é considerado como a macroalga mais importante em termos de abundância em costões rochosos do litoral sudeste brasileiro (Oliveira Filho \& Paula 1983, Paula \& Eston 1989). Széchy (1996) estudando bancos de Sargassum nos Estados do Rio de Janeiro e São Paulo, utiliza a biomassa de Sargassum

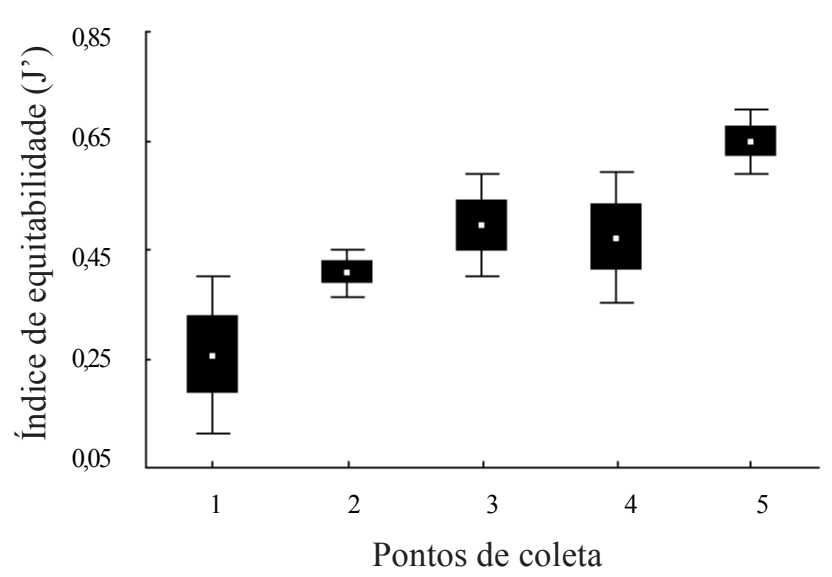

Figura 5. Representação gráfica dos valores do índice de equitabilidade $(\mathrm{J})$ calculados para os cinco locais estudados. - média \pm erro padrão; I média \pm desvio padrão; $\square$ média.

Figure 5. Eveness index $(\mathrm{J})$ calculated for the five studied sites. mean \pm standard error; I mean \pm standard deviation; $\square$ mean. 


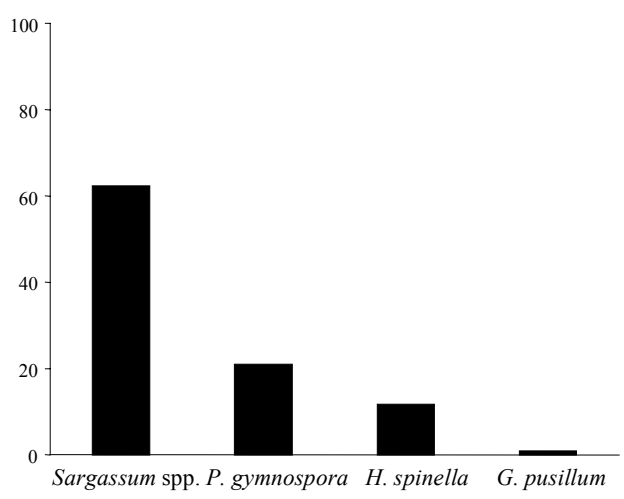

A
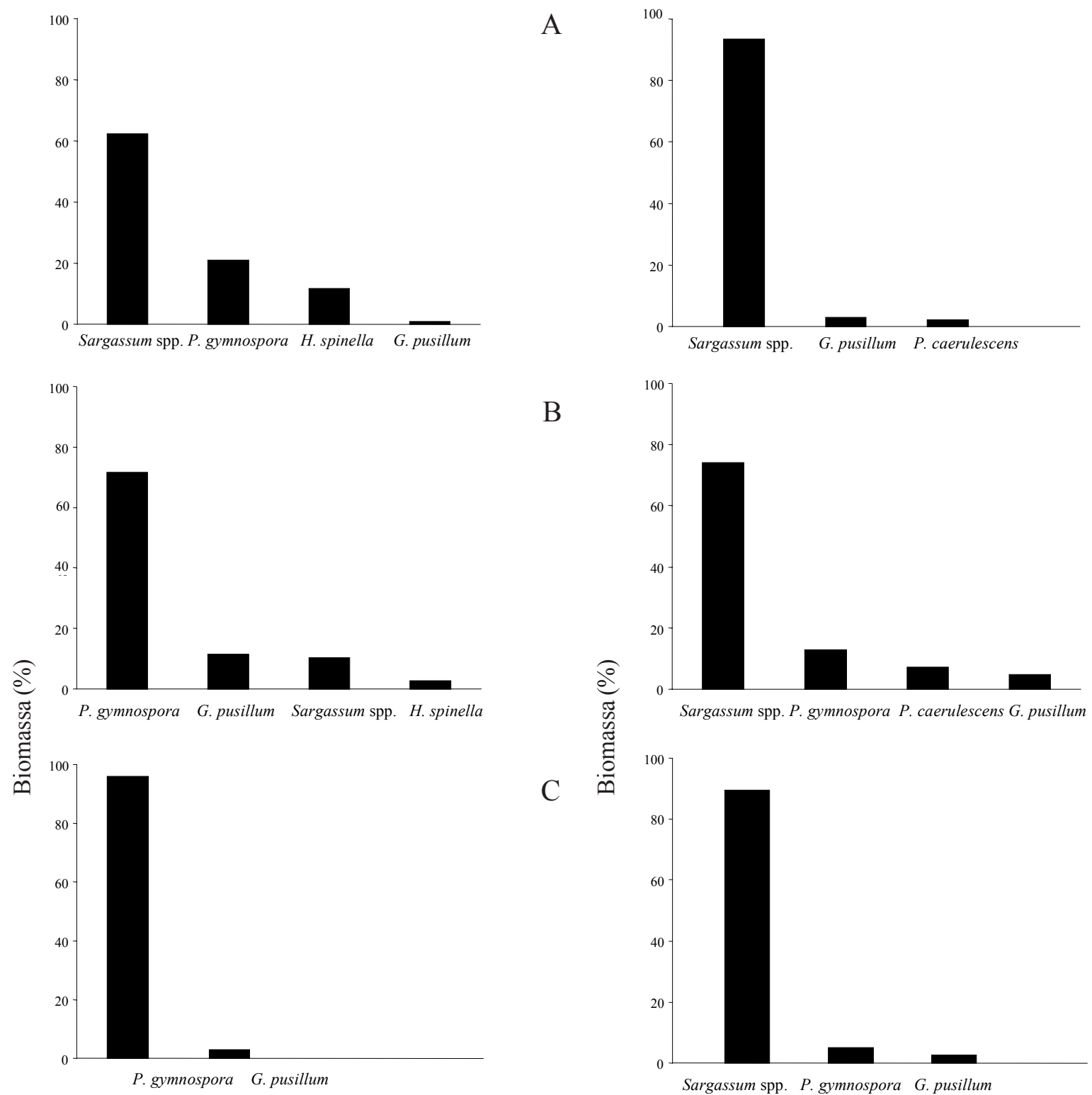

B

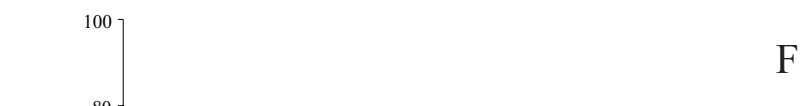

$\mathrm{C}$
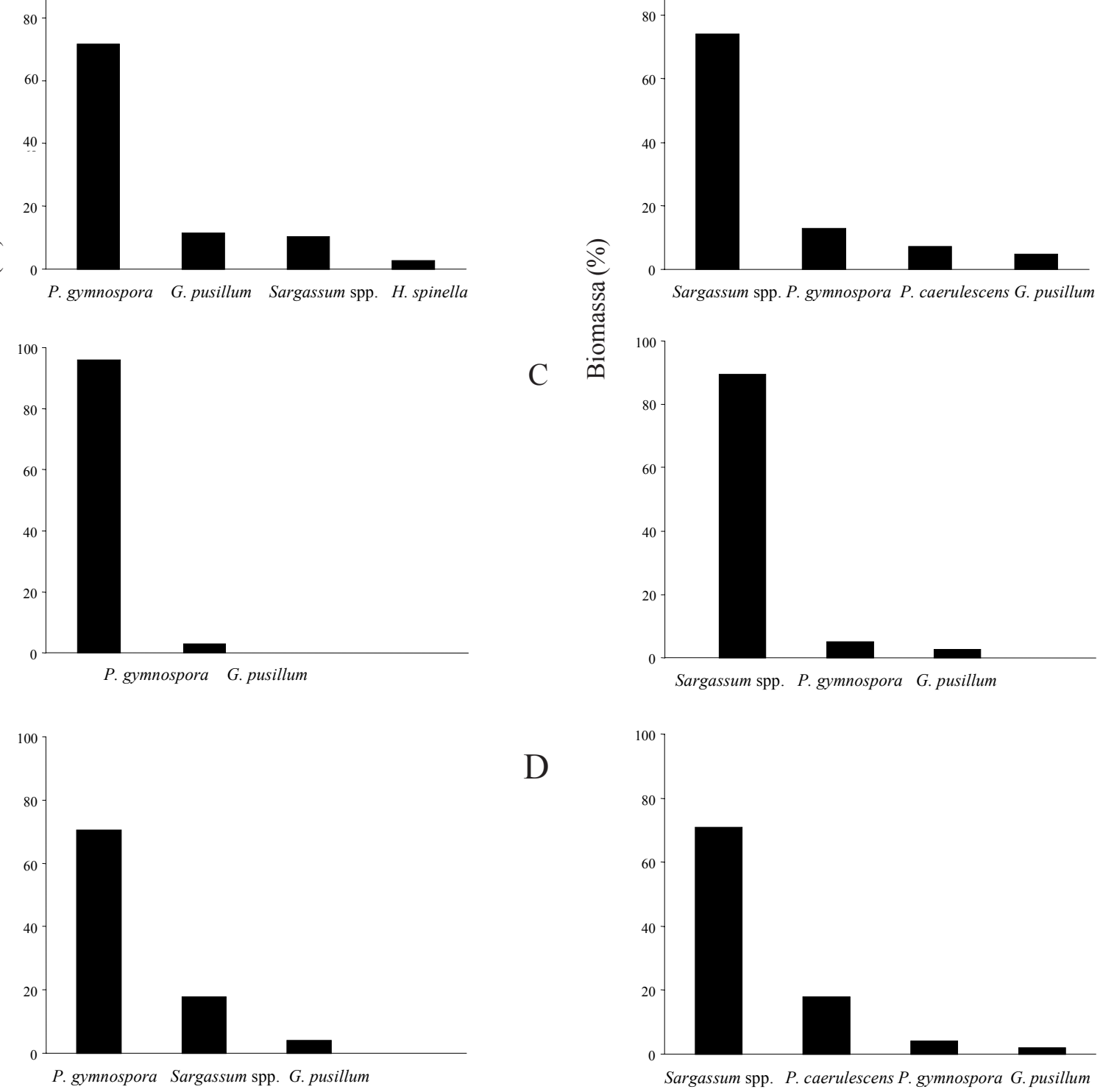

D

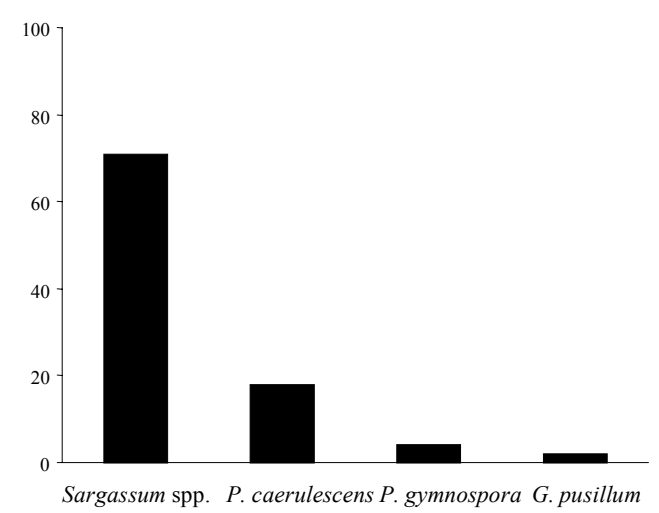

Táxons

Figura 6. Percentual de contribuição média da biomassa dos táxons mais representativos para a biomassa total dos pontos de coleta 1 (A - verão; B - outono; C - inverno; D - primavera) e 2 (E - verão; F - outono; G - inverno; H - primavera).

Figure 6. Biomass contribution of the most abundant taxa in relation to the total biomass from the sites 1 (A - Summer; B Autumn; C - Winter; D - Spring) and 2 (E - Summer; F - Autumn; G - Winter; H - Spring). 
Tabela 2. Biomassa média $\left(\mathrm{g} \cdot \mathrm{m}^{-2}\right.$ ) dos táxons encontrados nos cinco locais de coleta e que contribuíram com mais de $1 \%$ para a biomassa média total do local.

Table 2. Mean biomass $\left(\mathrm{g} . \mathrm{m}^{-2}\right.$ ) of taxa from the five studied sites that contributed with more than $1 \%$ for the mean total local biomass.

\begin{tabular}{|c|c|c|c|}
\hline Táxons/épocas do ano & g.m $\mathrm{m}^{-2}$ & Táxons/épocas do ano & g.m $\mathrm{m}^{-2}$ \\
\hline \multicolumn{4}{|l|}{ Ponto 1} \\
\hline VERÃO & & OUTONO & \\
\hline Hypnea spinella & 24,3 & Gelidium pusillum var. pusillum & 9,5 \\
\hline Padina gymnospora & 43,2 & Hypnea spinella & 2,2 \\
\hline Pterocladiella caerulescens & 6,5 & Padina gymnospora & 59,8 \\
\hline Sargassum spp. & 127,9 & Pterocladiella caerulescens & 2,1 \\
\hline INVERNO & & $\begin{array}{l}\text { Sargassum spp. } \\
\text { PRIMAVERA }\end{array}$ & 8,6 \\
\hline Pterocladiella caerulescens & 2,1 & Chondracanthus acicularis & 3,4 \\
\hline \multirow[t]{4}{*}{ Padina gymnospora } & 185,4 & Gelidium pusillum var. pusillum & 5,1 \\
\hline & & Hypnea spinella & 1,4 \\
\hline & & Padina gymnospora & 74,2 \\
\hline & & Sargassum spp. & 18,6 \\
\hline \multicolumn{4}{|l|}{ Ponto 2} \\
\hline VERÃO & & OUTONO & \\
\hline Gelidium pusillum var. pusillum & 6,5 & Gelidium pusillum var. pusillum & 5,3 \\
\hline Pterocladiella caerulescens & 4,6 & Padina gymnospora & 14,0 \\
\hline \multirow[t]{2}{*}{ Sargassum spp. } & 84,5 & Pterocladiella caerulescens & 7,9 \\
\hline & & Sargassum spp. & 80,6 \\
\hline INVERNO & & PRIMAVERA & \\
\hline Gelidium pusillum var. pusillum & 4,2 & Chondracanthus acicularis & 2,1 \\
\hline Hypnea spinella & 1,6 & Codium decorticatum & 3,7 \\
\hline Padina gymnospora & 7,6 & Gelidium pusillum var. pusillum & 2,9 \\
\hline \multirow[t]{3}{*}{ Sargassum spp. } & 34,7 & Hypnea spinella & 4,5 \\
\hline & & Pterocladiella caerulescens & 26,0 \\
\hline & & Sargassum spp. & 98,3 \\
\hline \multicolumn{4}{|l|}{ Ponto 3} \\
\hline VERÃO & & OUTONO & \\
\hline Codium taylorii & 4,1 & Gelidiopsis planicaulis & 24,6 \\
\hline Gelidiopsis planicaulis & 8,5 & Hypnea musciformis & 71,6 \\
\hline Hypnea musciformis & 60,3 & Hypnea spinella & 4,3 \\
\hline Padina gymnospora & 26,8 & Padina gymnospora & 16,5 \\
\hline Pterocladiella caerulescens & 3,5 & Pterocladiella caerulescens & 2,2 \\
\hline Sargassum spp. & 117,3 & Sargassum spp. & 92,7 \\
\hline INVERNO & & PRIMAVERA & \\
\hline Acanthophora spicifera & 5,6 & Chondracanthus teedei & 2,6 \\
\hline Codium decorticatum & 6,9 & Gelidiopsis planicaulis & 52,7 \\
\hline Gelidiopsis planicaulis & 17,8 & Gelidium pusillum var. pusillum & 7,2 \\
\hline Hypnea musciformis & 74,9 & Hypnea musciformis & 37,6 \\
\hline Jania adhaerens & 4,0 & Hypnea spinella & 9,4 \\
\hline Padina gymnospora & 6,1 & Padina gymnospora & 4,2 \\
\hline Pterocladiella caerulescens & 9,9 & Pterocladiella caerulescens & 26,7 \\
\hline \multirow[t]{2}{*}{ Sargassum spp. } & 218,1 & Sargassum spp. & 112,2 \\
\hline & & Ulva lactuca & 3,7 \\
\hline \multicolumn{4}{|l|}{ Ponto 4} \\
\hline VERÃO & & OUTONO & \\
\hline Chondracanthus acicularis & 1,4 & Acanthophora spicifera & 0,7 \\
\hline Codium decorticatum & 1,9 & Chondracanthus acicularis & 1,6 \\
\hline Gelidiopsis planicaulis & 3,7 & Gelidiopsis planicaulis & 4,0 \\
\hline
\end{tabular}


continuação

\begin{tabular}{|c|c|c|c|}
\hline Táxons/épocas do ano & g.m $\mathrm{m}^{-2}$ & Táxons/épocas do ano & g.m $\mathrm{m}^{-2}$ \\
\hline VERÃO (cont.) & & OUTONO (cont.) & \\
\hline Gelidiopsis variabilis & 3,5 & Gelidium pusillum var. pusillum & 0,8 \\
\hline Gelidium pusillum var. pusillum & 7,0 & Gracilaria cervicornis & 0,6 \\
\hline Gracilaria cervicornis & 3,4 & Hypnea spinella & 11,0 \\
\hline Hypnea spinella & 3,6 & Padina gymnospora & 12,3 \\
\hline Pterocladiella caerulescens & 1,2 & Pterocladiella caerulescens & 1,3 \\
\hline Sargassum spp. & 72,8 & Sargassum spp. & 15,4 \\
\hline INVERNO & & PRIMAVERA & \\
\hline Acanthophora spicifera & 0,4 & Acanthophora spicifera & 24,9 \\
\hline Champia vieillardii & 0,4 & Chondracanthus acicularis & 2,0 \\
\hline Chondracanthus acicularis & 0,8 & Codium taylorii & 3,2 \\
\hline Dictyota ciliolata & 5,3 & Gelidiopsis planicaulis & 5,6 \\
\hline Gelidiopsis planicaulis & 1,2 & Gelidium pusillum var. pusillum & 4,3 \\
\hline Gelidium crinale & 1,0 & Gracilaria cervicornis & 2,2 \\
\hline Gelidium pusillum var. pusillum & 3,9 & Hypnea spinella & 9,8 \\
\hline Hypnea spinella & 5,5 & Pterocladiella caerulescens & 2,4 \\
\hline Padina gymnospora & 7,6 & Sargassum spp. & 140,7 \\
\hline Pterocladiella caerulescens & 0,6 & & \\
\hline Sargassum spp. & 4,0 & & \\
\hline \multicolumn{4}{|l|}{ Ponto 5} \\
\hline VERÃO & & OUTONO & \\
\hline Caulerpa sertularioides & 69,6 & Caulerpa sertularioides & 239,9 \\
\hline Codium taylorii & 33,1 & Dictyopteris delicatula & 112,6 \\
\hline Dictyopteris delicatula & 45,9 & Galaxaura marginata & 5,8 \\
\hline Galaxaura marginata & 54,9 & Gelidiopsis planicaulis & 32,6 \\
\hline Gelidiopsis planicaulis & 16,6 & Gracilaria cervicornis & 90,8 \\
\hline Gracilaria cervicornis & 63,1 & Hypnea spinella & 11,8 \\
\hline Hypnea musciformis & 5,7 & Sargassum spp. & 20,2 \\
\hline Hypnea spinella & 52,0 & Spatoglossum schroederi & 28,4 \\
\hline Sargassum spp. & 79,0 & PRIMAVERA & \\
\hline Spatoglossum schroederi & 20,2 & Amphiroa beauvoisii & 13,5 \\
\hline INVERNO & & Centroceras clavulatum & 15,0 \\
\hline Chondracanthus acicularis & 6,1 & Chondracanthus teedei & 18,4 \\
\hline Chondracanthus teedei & 11,1 & Codium decorticatum & 33,7 \\
\hline Codium taylorii & 25,6 & Codium taylorii & 73,9 \\
\hline Dictyopteris delicatula & 57,5 & Colpomenia sinuosa & 7,5 \\
\hline Dictyota ciliolata & 11,1 & Dictyopteris delicatula & 77,2 \\
\hline Galaxaura marginata & 6,9 & Galaxaura marginata & 39,8 \\
\hline Gracilaria cervicornis & 36,4 & Gelidiopsis planicaulis & 34,2 \\
\hline Hypnea spinella & 17,2 & Gracilaria cervicornis & 56,2 \\
\hline Padina gymnospora & 27,5 & Hypnea musciformis & 20,6 \\
\hline Padina tetrastromatica & 4,2 & Hypnea spinella & 18,4 \\
\hline Sargassum spp. & 27,5 & Padina gymnospora & 149,2 \\
\hline Spatoglossum schroederi & 2,8 & Pterocladiella caerulescens & 7,5 \\
\hline \multirow[t]{5}{*}{ Ulva lactuca } & 4,1 & Rhodymenia pseudopalmata & 15,9 \\
\hline & & Sargassum spp. & 65,0 \\
\hline & & Solieria filiformis & 7,8 \\
\hline & & Spatoglossum schroederi & 25,6 \\
\hline & & Ulva lactuca & 29,3 \\
\hline
\end{tabular}



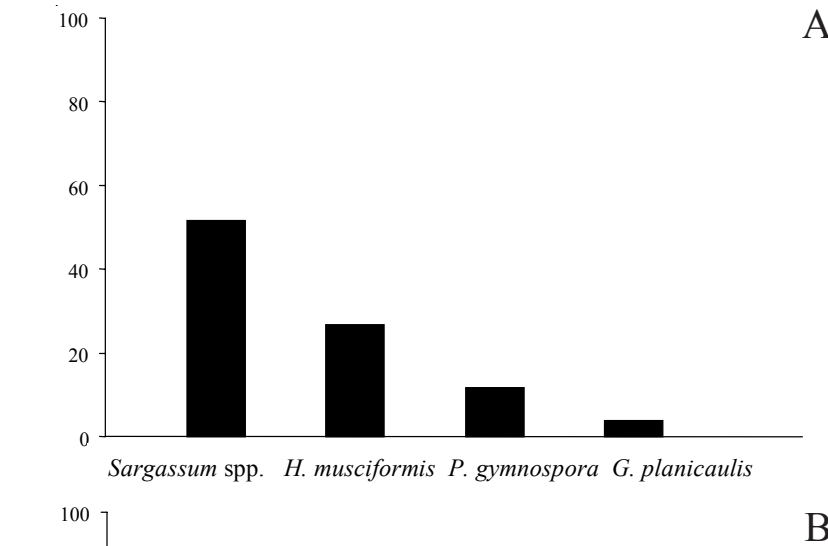

B

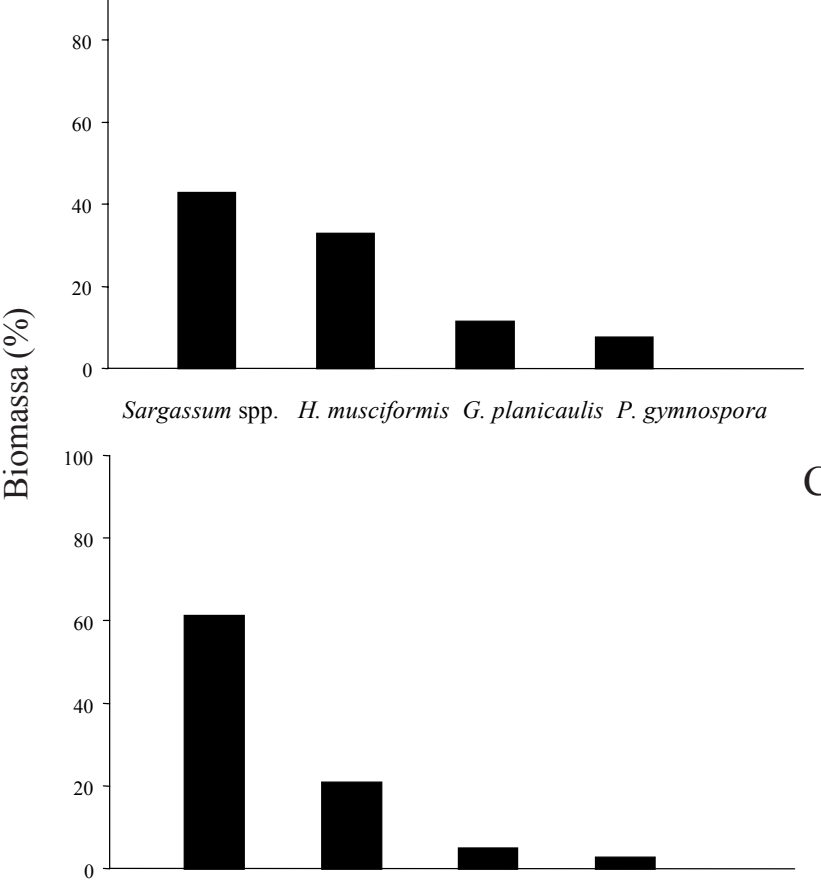

Sargassum spp. H. musciformis G. planicaulis P. caerulescens

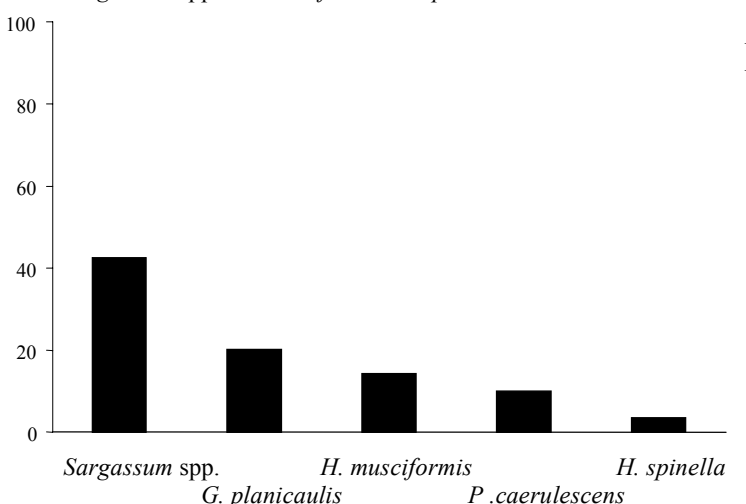

Táxons

Figura 7. Percentual de contribuição média da biomassa dos táxons mais representativos para a biomassa total do ponto de coleta 3. A - verão; B - outono; C - inverno; D - primavera.

Figure 7. Biomass contribution of the most abundant taxa in relation to the total biomass from the site 3 (A - Summer; B Autumn; C - Winter; D - Spring).

A

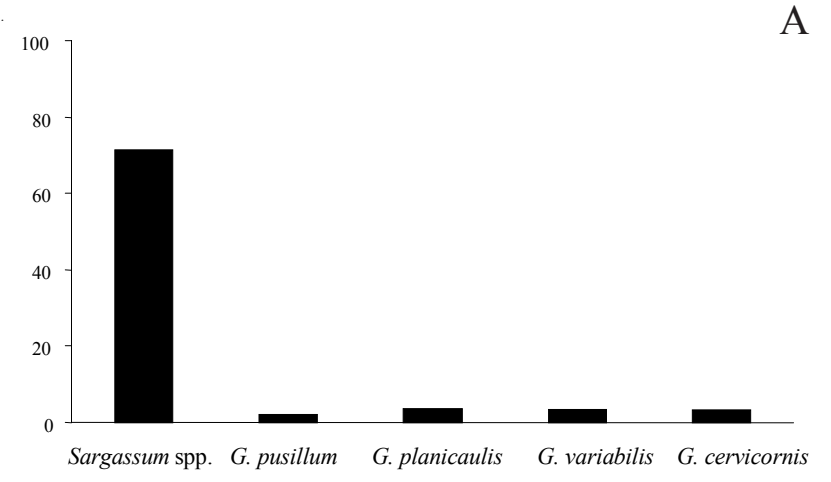

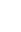
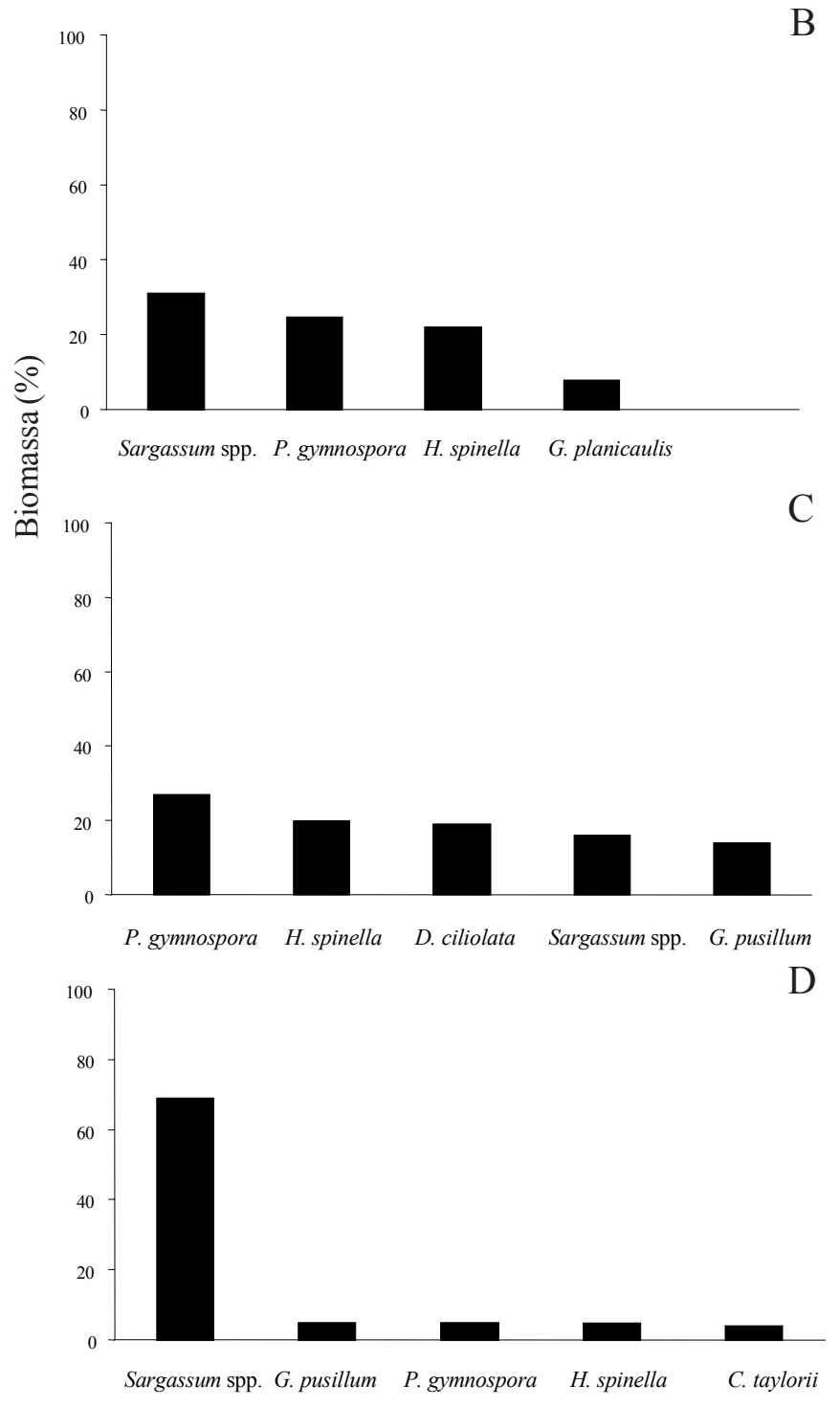

Táxons

Figura 8. Percentual de contribuição média da biomassa dos táxons mais representativos para a biomassa total do ponto de coleta 4. A - verão; B - outono; C - inverno; D - primavera.

Figure 8. Biomass contribution of the most abundant taxa in relation to the total biomass from the site 4 (A - Summer; B Autumn; C - Winter; D - Spring). 


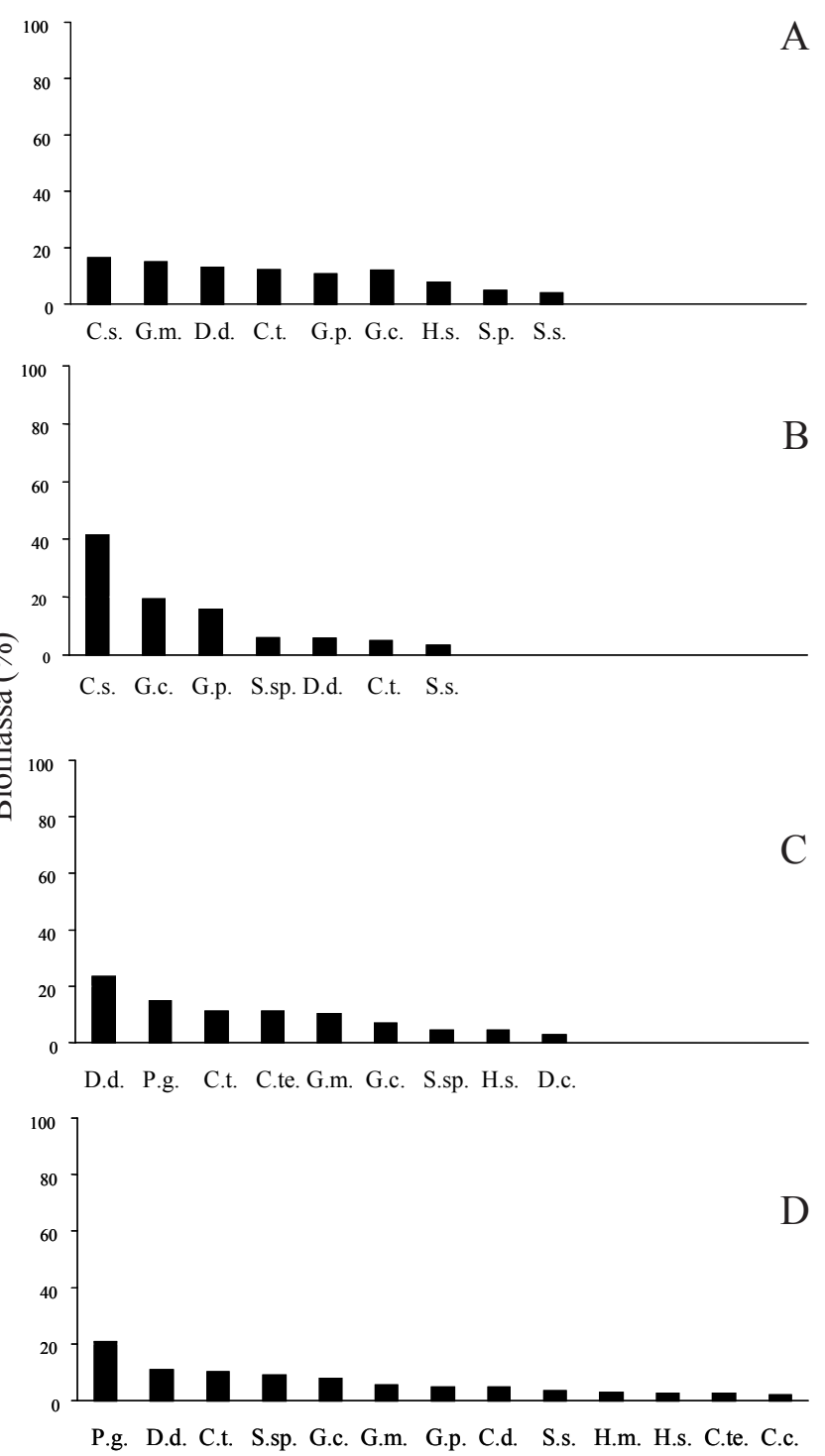

Táxons

Figura 9. Percentual de contribuição média da biomassa dos táxons mais representativos para a biomassa total do ponto de coleta 5. A - verão; B - outono; C - inverno; D - primavera. C.c. - C. clavulatum, C.d. - C. decorticatum, Cs. C. sertularioides, C.t. - C. taylorii, C.te. - C. teedei, D.c. D. ciliolata, D.d - D. delicatula, G.c. - G. cervicornis, G.m. G. marginata, G.p. - G. planicaulis, H.m. - H. musciformis, H.s. - H. spinella, P.g. - P. gymnospora, S.s. - S. schroederi, S.sp. - Sargassum spp.

Figure 9. Biomass contribution of the most abundant taxa in relation to the total biomass from the site 5 (A - Summer; B - Autumn; C - Winter; D - Spring). C.c. - C. clavulatum, C.d. - C. decorticatum, Cs. - C. sertularioides, C.t. - C. taylorii, C.te. - C. teedei, D.c. - D. ciliolata, D.d - D. delicatula, G.c. G. cervicornis, G.m. - G. marginata, G.p. - G. planicaulis, H.m. - H. musciformis, H.s. - H. spinella, P.g. - P. gymnospora, S.s. - S. schroederi, S.sp. - Sargassum spp. em cada local amostrado como um dos critérios principais para caracterizar o tipo de banco encontrado. Seguindo-se o critério estabelecido por Széchy (1996), os 5 locais amostrados na BS, enquadram-se no padrão " 2 b", comunidades onde Sargassum ocorre em pequena ou razoável quantidade $\left(66,0\right.$ g.m $\mathrm{m}^{-2}$ a $213,9 \mathrm{~g} \cdot \mathrm{m}^{-2}$ massa seca). Segundo Széchy (1996), este padrão é observado em comunidades de modo protegido e moderadamente protegido. Estas conclusões estão de acordo com o encontrado para os 5 pontos amostrados na BS, onde os pontos 1, 2 e 4 podem ser considerados protegidos e os pontos 3 e 5 como moderadamente protegidos da ação das ondas.

Além de Sargassum spp., as populações de P. gymnospora também apresentaram percentual de contribuição importante. A ocorrência de P. gymnospora. como uma das espécies mais abundantes associadas aos bancos de Sargassum tem sido reconhecida tanto para a região sudeste (Oliveira Filho \& Paula 1983, Széchy 1996, Reis \& YoneshigueValentin 1998) como para a região nordeste (Eston et al. 1986, Amado Filho et al. 1997b) do litoral brasileiro. Outras espécies, de menor porte, como Hypnea spp., G. pusillum, P. caerulescens e Gelidiopsis spp., que contribuíram com mais de $1 \%$ para a biomassa média total nos 5 pontos coletados, foram referidas por Széchy (1996) como espécies importantes quanto a cobertura e/ou biomassa para bancos de Sargassum nos Estados do Rio de Janeiro e São Paulo. Comparando-se os percentuais de contribuição das principais espécies do ponto 3 com os resultados obtidos por Reis \& Yoneshigue-Valentin (1998) para o mesmo local entre os anos de 1994 e 1996, pôde-se verificar que a estrutura da comunidade deste local se manteve semelhante no período 1994-1999, no qual Sargassum spp., P. gymnospora e H. musciformis contribuem juntas com pelo menos $60 \%$ para a biomassa do local em ambos os estudos.

Sabino \& Villaça (1999) comparam métodos de amostragem de comunidades de costão e comentam que atualmente a preocupação em determinar as mudanças na estrutura das comunidades, devido aos impactos de ações antrópicas no meio ambiente marinho requer técnicas mais ágeis de amostragem, e que apesar da técnica de raspagem e posterior análise qualiquantitativa do material contido nos quadrados ser uma das mais completas, é uma técnica trabalhosa e demorada. Entretanto, a escolha neste trabalho do método de amostragem destrutivo, permitiu uma caracterização detalhada da composição e da estrutura das comunidades fitobentônicas da BS, o que facilitará 
no futuro, a identificação de eventuais mudanças através de abordagens metodológicas mais simples.

Agradecimentos - Ao Programa Pibic/CNPq do JBRJ que concedeu as bolsas de iniciação científica para as alunas Bianca V. Marins e Claudia Felix, sem as quais esse trabalho não seria possível. Trabalho executado com apoio financeiro do CNPq (521688/96-5) e Faperj (E-26/170.336/98).

\section{Referências bibliográficas}

AMADO FILHO, G.M., ANDRADE, L.R., KAREZ, C.S., YONESHIGUE-VALENTIN, Y. \& PFEIFFER, W.C. 1997a. Effects on growth and accumulation of zinc in six seaweed species. Ecotoxicology and Environmental Safety 37:223-228.

AMADO FILHO, G.M., ANDRADE, L.R., REIS, R.P., BASTOS, W. \& PFEIFFER, W.C. 1997b. Heavy metal concentration in seaweed species from the Abrolhos reef region, Brazil. Proceedings of the $8^{\text {th }}$ International Coral Reef Symposium 2:1843-1846.

AMADO FILHO, G.M., REZENDE, C.E. \& LACERDA, L.D. 1999a. Poluição da baía de Sepetiba já ameaça outras áreas. Ciência Hoje 25:46-48.

AMADO FILHO, G.M., ANDRADE, L.R., KAREZ, C.S., FARINA, M. \& PFEIFFER, W.C. 1999b. Brown algae species as biomonitors of $\mathrm{Zn}$ and $\mathrm{Cd}$ at Sepetiba Bay, Rio de Janeiro, Brazil. Marine Environmental Research 48:213- 224.

ARAÚJO, F.G., CRUZ FILHO, A.G., AZEVÊDO, M.C.C., SANTOS, A.C.A. \& FERNANDES, L.A.M. 1997. Estrutura da Comunidade de Peixes Jovens da Margem Continental da Baía de Sepetiba, RJ. Acta Biológica Leopoldensia 19:61-83.

ARAÚJO, F.G., GOMES, I.D. \& BERTOLDO, I.C. 1999. Proporção sexual e período reprodutivo de três espécies de Gerreidae (Osteichthyes, Perciformes) na Baía de Sepetiba, RJ. Revista Brasileira de Medicina Veterinária 21:207-210.

BARCELLOS, C. 1995. Geodinâmica do cádmio e zinco na baía de Sepetiba. Tese de doutorado, Universidade Federal Fluminense, Rio de Janeiro.

BERCHEZ, F.A.S. \& OLIVEIRA, E.C. 1992. Temporal changes in the benthic marine flora of Baía de Santos, SP, Brazil, over the last four decades. In Algae and environment: a general approach (M. Cordeiro-Marino, M.T.P. Azevedo, C.L. Sant'Anna, N. Yamaguishi-Tomita \& E.M. Plastino, eds.). Sociedade Brasileira de Ficologia, São Paulo, p.120-131.

BROWER, J.E., ZAR, J.H. \& VON ENDE, C. 1997. Field and laboratory methods for general ecology. McGraw-Hill, New York.

ENGDAHL, S., MAMBOYA, F., MTOLERA, M., SEMESI, A. \& BJORK, M. 1998. The brown macroalgae Padina boergesenii as an indicator of heavy metal contamination in the Zanzibar Chanel. Ambio 27:694-700.
ESTON, V.R., MIGOTTO, A.E., OLIVEIRA FILHO, E.C., RODRIGUES, S.A. \& FREITAS, C. 1986. Vertical distribution of benthic marine organisms on rocky coasts of the Fernando de Noronha Archipelago (Brazil). Boletim do Instituto Oceanografico 34:37-53.

FEEMA/GTZ 2000. Avaliação da qualidade da água da Bacia da Baía de Sepetiba.Edu@Web editora, CD-ROM.

HEWITT, C.L. \& MARTIN, R.B. 2001. Revised protocols for baseline port surveys for introduced marine species - survey design, sampling protocols and specimen handling. Technical Report 22, CSIRO Marine Research, Hobart.

JAENICKE, L. 1977. Sex hormones of brown algae. Naturwissenschaften 64:69-75

LUBCHENCO, J., OLSON, A.M., BRUBAKER, L.B., CARPENTER, S.R., HOLLAND, M.N., HUBBELL, S.P., LEVIN, S.A., MACMAHON, J.A., MATSON, P.A., MELILLO, J.M., MOONEY, H.A., PETERSON, C.H., PULLIAM, H.R., REAL, L.A., REGAL, P.J. \& RISSER, P.G. 1991. The sustainable biosphere initiative: an ecological research agenda. Ecology 72:371-412.

MARKHAM, J., KREMER, B.P. \& SPERLING, K.R. 1980. Effects of cadmium on Laminaria saccharina in culture. Marine Ecology Progress Series 3:31-39.

MITCHELL, G.J., SZÉCHY, M.T.M. \& MITSUYA, L.A 1979. Sinopse das clorofíceas marinhas bentônicas do litoral do estado do Rio de Janeiro. Leandra 8-9:91-123.

MURUGADAS, T.L., PHANG, S.M. \& TONG, S.L. 1995. Heavy metal accumulation patterns in selected seaweed species of Malaysia. Asia-Pacific Journal of Molecular Biology and Biothechnology 3:209-310.

OLIVEIRA FILHO, E.C. \& PAULA, E.J. 1983. Aspectos da distribuição vertical e variação sazonal de comunidades da zona de marés em costões rochosos do litoral norte do Estado de São Paulo. Publicações Instituto de Pesquisas da Marinha 147:44-71.

PAULA, E.J. \& ESTON, V.R. 1989. Secondary succession on an exposed rocky intertidal algal community of the state of São Paulo (Brazil). Boletim de Botânica da Universidade de São Paulo 11:1-9.

PEDRINI, A.G. 1980. Algas marinhas bentônicas da Baía de Sepetiba e arredores (Rio de Janeiro). Dissertação de mestrado, Universidade Federal do Rio de Janeiro, Rio de Janeiro.

REES, J.G., BREWARD, N., MADDOCK, J.E.L., FERGUSON, A.J. \& WILLIAMS, T.M. 1998 Land ocean contamination study (LOCS): assessment of contamination by metals and selected organic compounds in coastal sediments and waters of Sepetiba Bay, Rio de Janeiro State, Brazil. Technical Report WC/ 98/30, Overseas Geology Series, British Geological Survey, Nottingham. 
REIS, R.P. \& YONESHIGUE-VALENTIN,Y. 1998. Variação espaço-temporal de populações de Hypnea musciformis (Rhodophyta, Gigartinales) na Baía de Sepetiba e Armação dos Búzios, RJ, Brasil. Acta Botanica Brasilica 12:465-483.

SABINO, C.M. \& VILLAÇA, R. 1999. Estudo comparativo de métodos de amostragem de comunidades de costão. Revista Brasileira de Biologia 59:407-419.

SZÉCHY, M.T.M. 1996. Estrutura de bancos de Sargassum (Phaeophyta- Fucales) do litoral dos estados do Rio de Janeiro e São Paulo. Tese de doutorado, Universidade de São Paulo, São Paulo.
TEIXEIRA, V.L., PEREIRA, R.C., MARQUES JUNIOR, A.N., LEITÃO FILHO, C.M. \& SILVA, C.A.R. 1987. Seasonal variations in infralittoral seaweed communities under a pollution gradient in baía de Guanabara, Rio de Janeiro (Brazil). Ciência \& Cultura 39:423-428.

UNDERWOOD, A.J. 1981. Techniques of analysis of variance in experimental marine biology and ecology. Oceanography and Marine Biology Annual Review 19:513-605.

WINER, B.J. 1971. Statistical principles in experimental design. $2^{\text {nd }}$ ed. McGraw-Hill, Tokyo.

ZAR, J.H. 1996. Biostatistical analysis. Prentice-Hall, New Jersey. 